\title{
A dominant acoustic-gravity mode in the polar thermosphere
}

\author{
A. K. Fedorenko, A. V. Bespalova, O. K. Cheremnykh, and E. I. Kryuchkov \\ Space Research Institute of NAS Ukraine and SSA Ukraine, Prosp. Akad. Glushkova, 40, build. 4/1, \\ 03680 MSP, Kyiv 187, Ukraine \\ Correspondence to: A. K. Fedorenko (aurora28@i.ua)
}

Received: 2 June 2014 - Revised: 6 November 2014 - Accepted: 19 December 2014 - Published: 27 January 2015

\begin{abstract}
The article presents a summary of the main findings of the systematic study of acoustic-gravity waves (AGWs) in the polar thermosphere. This study was based on the in situ measurements made by the Dynamics Explorer 2 (DE2) spacecraft late in its mission when it descended low enough $(250-400 \mathrm{~km})$. It was found out that AGWs in the polar thermosphere are observed within a narrow frequency band close to the Brunt-Väisälä frequency and with horizontal wavelengths about $500-600 \mathrm{~km}$. The broadband spectrum of travelling ionospheric disturbance (TID) frequencies observed by radars is caused by the Doppler effect. The AGW amplitudes do not depend on the altitude, but grow almost linearly with the wind velocity. They propagate towards the wind.
\end{abstract}

Keywords. Ionosphere (ionosphere-atmosphere interactions) - meteorology and atmospheric dynamics (thermospheric dynamics; waves and tides)

\section{Introduction}

The polar regions host the most powerful sources of upper atmosphere perturbations. These sources are caused by the inflow of energy from the solar wind and the magnetosphere. For this reason, high-amplitude waves are often observed in the polar thermosphere. Among different types of waves an important role is being played by acoustic-gravity waves (AGWs), which transport the energy between different altitude layers and geographic regions. They generate an ionospheric response known as travelling ionospheric disturbance (TID), which can be located from the ground (Hines, 1960), and which cause problems for the transionospheric radio communications, especially for the global navigation satellite system (GNSS) signals (Arbesser-Rastburg and Jakowski, 2007; Förster and Jakowski, 2000). This type of wave was studied experimentally for more than half-acentury mainly with remote sensing methods and, to a much lesser extent, with in situ spacecraft measurements. However, the relation between AGWs and TIDs is far from being straightforward due to the influence of the geomagnetic field and particle precipitation. In the $\mathrm{F}$ region of ionosphere, the amplitude and phase relations between the AGW and the TID depends on the altitude distribution of the plasma density. In the polar region, where there are particle precipitations and field-aligned currents, the height profile of the plasma becomes unstable. Moreover in the F region, where the frequency of neutral-ion collisions is small compared to the gyrofrequency, the ion motion perpendicular to the magnetic field is inhibited. Therefore, along the geomagnetic field, which is almost vertical at high latitudes, the ions move together with the neutral particles. Across the field the ion motion appears due to the electromagnetic drift. These factors complicate the relationship between the AGW and the TID. In addition, ground-based data are subject to Doppler shifting due to high wind velocity, which reaches $1 \mathrm{~km} \mathrm{~s}^{-1}$ in the polar thermosphere (Cowling et al., 1971; Crowley et al., 1989; Vincent and Eckermann, 1990). Thus, it is necessary to combine ground-based and space-based measurements to get a clear picture of waves in the polar thermosphere.

Unfortunately, there are only a small number of space missions involving direct measurements of atmospheric parameters at ionospheric altitudes. The latest of such missions NASA's Atmospheric Explorer and Dynamic Explorer missions - were launched in 1970s. After that, only gravimetric spacecraft like CHAMP, GRACE, and GOCE have flown at such altitudes. Unfortunately, their accelerometer data are not suitable for the study of AGWs, since it is impossible to distinguish between different reasons of spacecraft acceleration without using models based on prior assumptions ( $\mathrm{S}$. Bruinsma, private communication, 2013). Even a spacecraft 

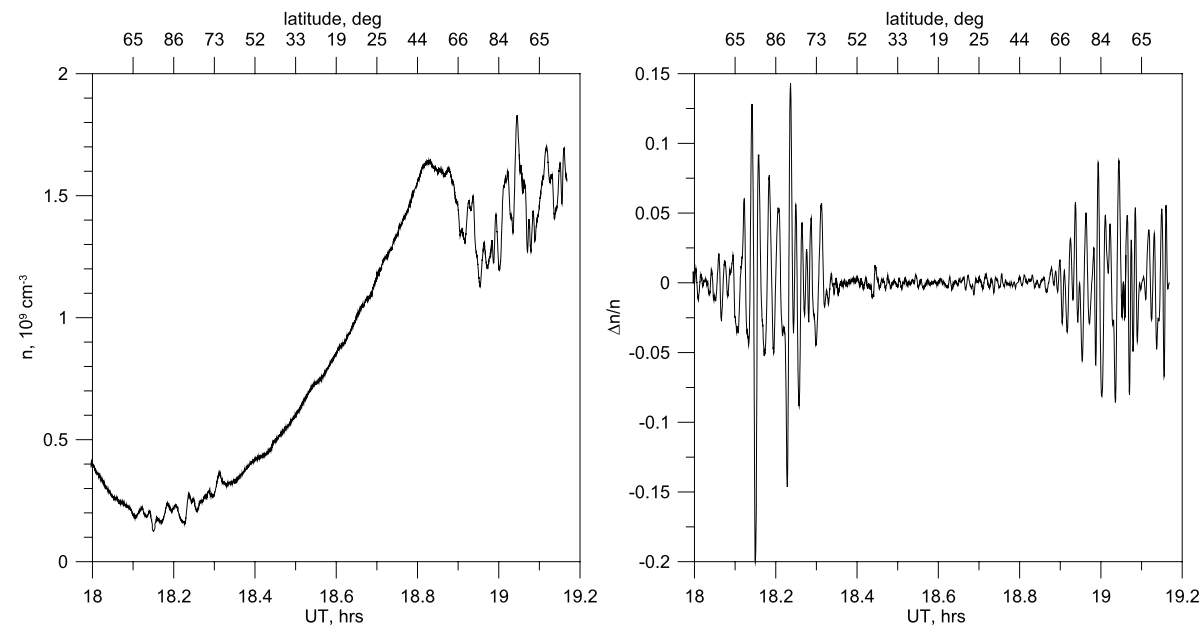

Figure 1. Neutral particle density (left) and relative density variation (right) along DE2 orbit 8257 (29 January 1983).

with a perfect instrument suite would be unable to measure all the parameters because its orbital velocity is much larger than the AGW phase velocity. For this reason, spacecraft actually observe snapshots of the waveform's projection along the orbit, i.e. spatial variations. Ground-based observations, on the other hand, capture temporal variations. This makes the comparison of the same events observed by spacecraft and ground-based stations very difficult. An ideal solution to this problem would be brought by a constellation of ionospheric spacecrafts. Nevertheless, it is possible to estimate many AGW parameters, such as the period, horizontal phase velocity, direction of propagation etc., using single spacecraft measurements (Fedorenko, 2009).

\section{Properties of polar AGWs}

It follows from the analysis of Dynamics Explorer 2 (DE2) data that at heights from 250 to $400 \mathrm{~km}$ above the polar caps the AGWs with wavelengths of several hundreds of kilometres are systematically observed at different geomagnetic activity levels (Fedorenko and Kryuchkov, 2011a). DE2's orbit with the perigee $250 \mathrm{~km}$, the apogee $1000 \mathrm{~km}$, and the inclination $89.9^{\circ}$ is very suitable for the study of polar waves. DE2's scientific payload included both neutral and charged components sensors, which allowed analysing the AGW/TID coupling directly (Carignan et al., 1981; Spencer et al., 1981). The typical distribution of the total density along the orbit is shown in Fig. 1 on the left.

Over the polar region, there are distinct wave variations registered, which are superimposed on the large-scale behaviour of parameters related to changes in the orbital height, large-scale dynamics, diurnal behaviour, etc. The wave-like processes were identified against large-scale changes in the parameters using the sliding average procedure. The number of points of the sliding window was selected to provide the maximum amount of cross-correlation between variations in the densities of different gases (Fedorenko, 2010). For the comparison of AGWs in various gases, one should consider relative variations of the densities normalized to an undisturbed average value. The relative variation of neutral density along the same orbit is shown in Fig. 1 on the right. AGW amplitudes in the polar regions reach 5-10\%, thus exceeding the amplitudes in mid and low latitudes by an order of magnitude. In the day sector the region of increased wave activity is bounded by the auroral oval, but in the night sector it can span down to lower geomagnetic latitudes. The dominant horizontal wavelength in the polar thermosphere is 500-600 km (Fedorenko and Kryuchkov, 2011a; Johnson et al., 1995). In the following subsections we briefly summarize the main features of polar AGWs as deduced primarily from DE2 measurements.

\subsection{Observational signatures}

To identify the type of the observed wave, its parameters should be compared to the theoretical predictions. As an example in Fig. 2 we plotted relative variations of densities of individual gases above the pole. The increase of the amplitude with the growth of the molecular mass (on the right) and the quasi-antiphase oscillations of $\mathrm{He}$ and heavy gases (on the left) are the characteristic signatures of AGW (Dudis and Reber, 1976; Fedorenko and Kryuchkov, 2011a).

The AGW variation of the density, observed from a satellite, is the result of the superposition of several factors: (1) the longitudinal wave compression due to the pressure gradient; (2) the adiabatic expansion/contraction of the gas; (3) the change in the background density at the vertical displacement of the volume under the influence of gravity. The difference between oscillation profiles in gases is caused by the difference in the vertical distribution of their density above the turbopause. In the observed variations of heavy 

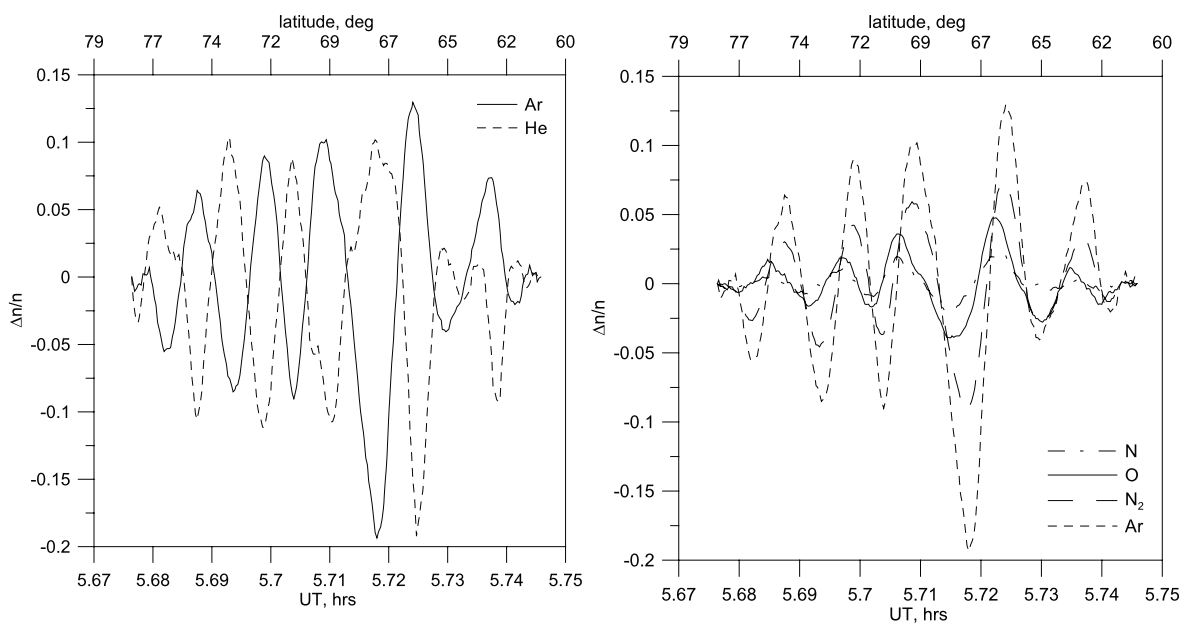

Figure 2. Relative density variation of $\mathrm{Ar}$ and $\mathrm{He}$ (left) and $\mathrm{N}, \mathrm{O}, \mathrm{N}_{2}$, and $\mathrm{Ar}$ (right) above the pole.

gases $\mathrm{N}_{2}(28 \mathrm{amu})$ and $\operatorname{Ar}(40 \mathrm{amu})$, the changes of the background density dominates. The wave variations of light gas He ( $4 \mathrm{amu})$ are caused mainly by the adiabatic expansion/contraction. During the maximal vertical displacement of the gas volume upwards, it expands adiabatically, so there is a minimum of He density. However, this extension is not enough to compensate the background gradient densities of heavy gases, and these gases demonstrate a maximum in the density. At the maximal vertical displacement of the gas downwards, there is a maximum of He density and a minimum of $\mathrm{N}_{2}$ and Ar densities. For gases $\mathrm{O}(16 \mathrm{amu})$ and $\mathrm{N}$ (14 amu) the adiabatic expansion/contraction is almost compensated by the change in background density and the elastic compression prevails in the resulting variations of the density. For this reason, the density variations of $\mathrm{O}$ and $\mathrm{N}$ are shifted in phase with respect to densities of $\mathrm{N}_{2}$ and $\mathrm{Ar}$, and their resulting amplitudes are small. The details of these features are described earlier in Dudis and Reber (1976) and Fedorenko (2009).

To check if the wave propagates in space it is necessary to study the relation between the vertical velocity $v_{z}$ and the vertical displacement $h$ of an elementary volume due to the wave. For a propagating monochromatic wave

$v_{z}=i \omega h$,

and the variations of $v_{z}$ are ahead of $h$ by $\pi / 2$ in the direction of propagation (Johnson et al., 1995). The vertical velocity $v_{z}$ was measured by the WATS experiment onboard DE2 (Spencer et al., 1981). The vertical displacement $h$ can be calculated by the relative variations of densities of two gases using a formula (Fedorenko, 2009)

$\frac{h}{H}=\frac{m}{m_{1}-m_{2}}\left(\frac{\delta n_{1}}{n_{1}}-\frac{\delta n_{2}}{n_{2}}\right)$,

where $n_{1}, n_{2}$ are the densities of the gases, $\delta n_{1}, \delta n_{2}$ are their absolute variations, $H=k T_{n} / m g$ is the height scale, $k$ is the
Boltzmann constant, $T_{n}$ is the neutral temperature, $m$ is the mean molecular mass, $m_{1}, m_{2}$ are the molecular masses of the gases, $g$ is the gravitational acceleration.

Figure 3 shows a typical example of the variations of $v_{z}$ and $h$ above the polar cap. It is easy to see that the wave propagates in reverse flight direction (leftwards in the plot). Almost all observed polar AGWs were propagating towards the dayside (Johnson et al., 1995; Fedorenko and Kryuchkov, 2011a). Note that the systematic phase shift between the density variation of different gases in Fig. 2 also indicates that the wave is propagating in space. A positive phase shift between the variations of $\mathrm{O}$ and $\mathrm{N}$ densities and the densities of heavier gases like $\mathrm{N}_{2}$ and $\mathrm{Ar}$ indicates the direction of wave propagation (Fedorenko, 2009).

Thus we conclude that the perturbations observed above the polar caps can be identified as propagating AGWs.

\subsection{Intrinsic frequency}

It is necessary to determine the AGW frequency from in situ measurements to compare them to the ground-based TID observations. The easiest way to do this is to use the relation (Eq. 1) between the amplitudes of the variations of $v_{z}$ and $h$. In Fig. 4 they are plotted against each other for seven different orbits. We chose the orbital segments above the polar caps in quiet conditions within the 250 $280 \mathrm{~km}$ altitude range. Two of them are in the northern hemisphere, and five are in the southern one. All these orbits show approximately the same dependence, which means that the corresponding frequencies are very close to each other. A linear fit to these dependencies gives the mean frequency $\omega \approx 9.8 \times 10^{-3} \mathrm{~s}^{-1}$, which corresponds to a period $T \approx 640 \mathrm{~s}$. The sound velocity in these conditions is about $c_{\mathrm{s}} \approx 850 \mathrm{~m} \mathrm{~s}^{-1}$, and the isothermic Brunt-Väisälä frequency $\omega_{\mathrm{b}}=g \sqrt{\gamma-1} / c_{\mathrm{s}} \approx 9.1 \times 10^{-3} \mathrm{~s}^{-1}$, where $\gamma$ is the ratio of specific heats. Within the estimation accuracy $\omega$ is close to 


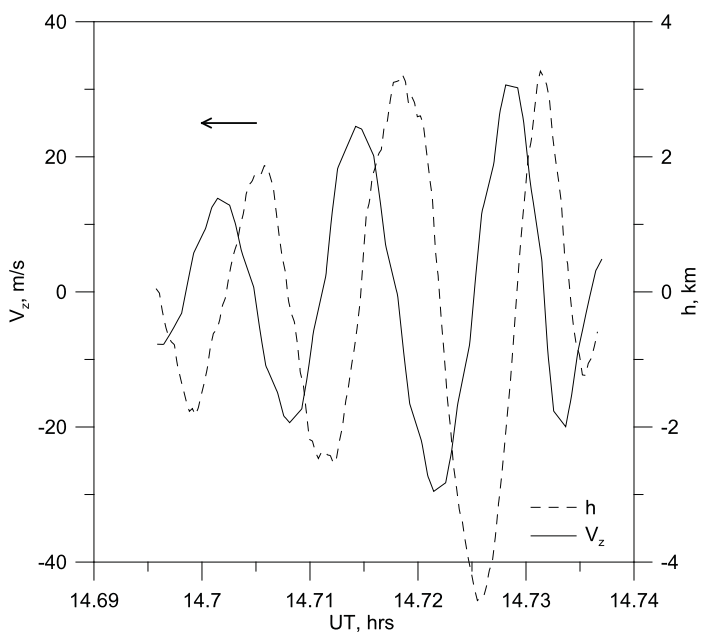

Figure 3. Variations of vertical velocity and displacement on the southern polar segment of the DE2 orbit 8286. The arrow indicates the direction of wave propagation.

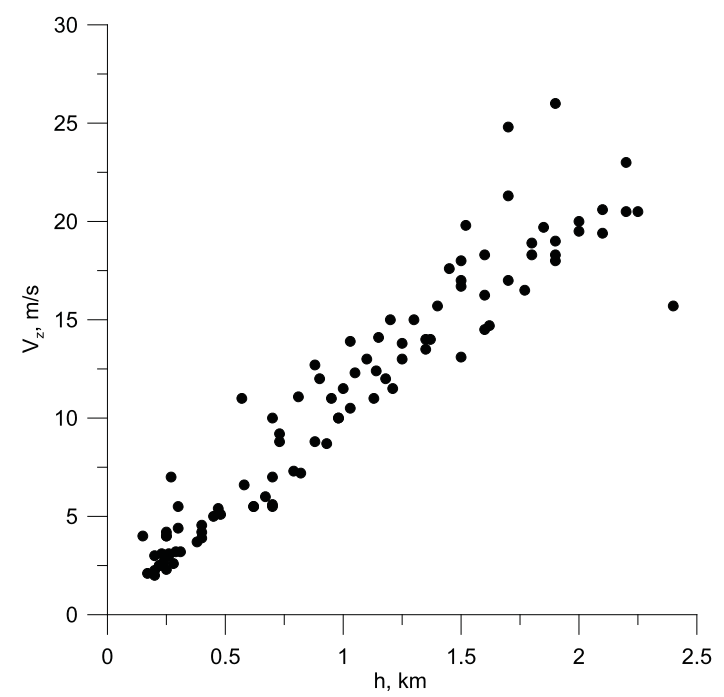

Figure 4. Amplitudes of variations of vertical velocity and displacement in different orbits.

$\omega_{\mathrm{b}}$ or slightly exceeds it. Note that $\omega$ is the intrinsic AGW frequency.

\subsection{Horizontal phase velocity}

The horizontal phase velocity $u_{x}$ can be estimated from the measured horizontal wavelength $\lambda_{x \mathrm{~s}}=2 \pi / k_{x \mathrm{~s}}$, which is a projection of the actual horizontal wavelength $\lambda_{x}$ onto the orbit, and the AGW period $T$. For the dominant values $\lambda_{x \mathrm{~s}}=$ $500-600 \mathrm{~km}$ and $T \approx(650 \pm 50) \mathrm{s}$ the estimated horizontal phase velocity equals $u_{x \mathrm{~s}}=\lambda_{x \mathrm{~s}} / T \approx 770-920 \mathrm{~m} \mathrm{~s}^{-1}$, which is close to the speed of sound at given conditions. Of course, AGWs can never become supersonic (Hines, 1960). In fact, it is always overestimated due to the projected wavelength

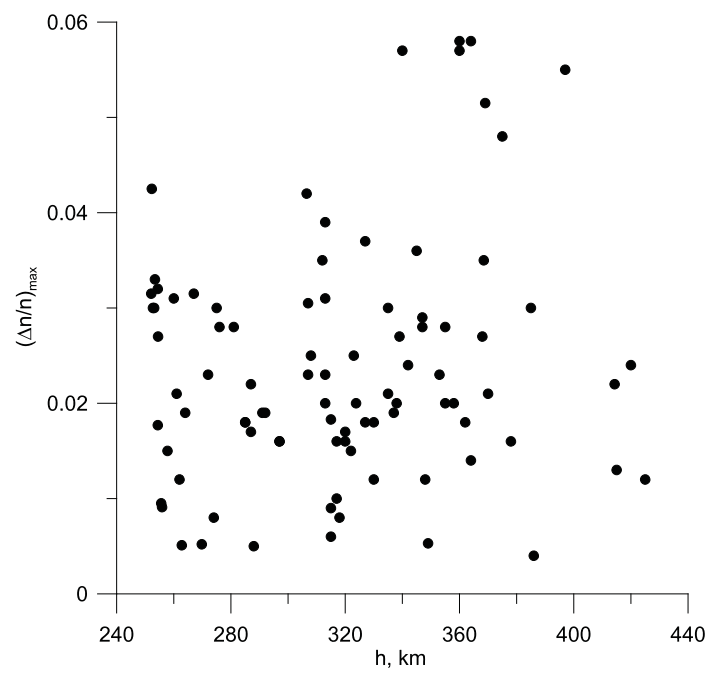

Figure 5. AGW amplitudes at different altitudes according to DE2 measurements.

being always larger than the actual one, $\lambda_{x s}>\lambda_{x}$. The actual coefficient depends on the angle between the wavefront and the orbit plane.

\subsection{No altitude dependence}

In the altitude range from 250 to $400 \mathrm{~km}$ no altitude dependence of AGW amplitude was observed (see Fig. 5). Each point on Fig. 5 corresponds to a maximum amplitude of a separate wave train. The classical AGW theory (Hines, 1960) predicts an exponential growth of the AGW amplitude as it propagates upwards due to the conservation of the wave energy in the atmosphere with the exponentially decreasing background density. In the real atmosphere above about $200 \mathrm{~km}$, the wave energy losses increase sharply due to the viscosity, especially when the mean free path of the particles becomes comparable with the wavelength. Therefore, in the real atmosphere, the increase in the amplitude of the AGW with height becomes slower. It is possible that the energy losses due to the molecular viscosity compensate the increase in the amplitude of the AGW. Another possible explanation is that AGWs propagate quasi-horizontally.

\subsection{AGW energy}

At altitudes between 250 and $400 \mathrm{~km}$ polar AGWs create vertical flows of energy up to $0.1 \mathrm{erg} \mathrm{cm}^{-2} \mathrm{~s}^{-1}$ (Fedorenko, 2010). This value is comparable to the energy brought by the precipitating particles in quiet geomagnetic conditions.

In AGW, in addition to the potential energy of acoustic compression, there is another kind of potential energy - thermobaric or gravitational potential energy associated with the vertical displacement of the gas volume under the action of the gravity. Polar AGWs also have a peculiar property: their acoustic and gravitational energy densities are almost 

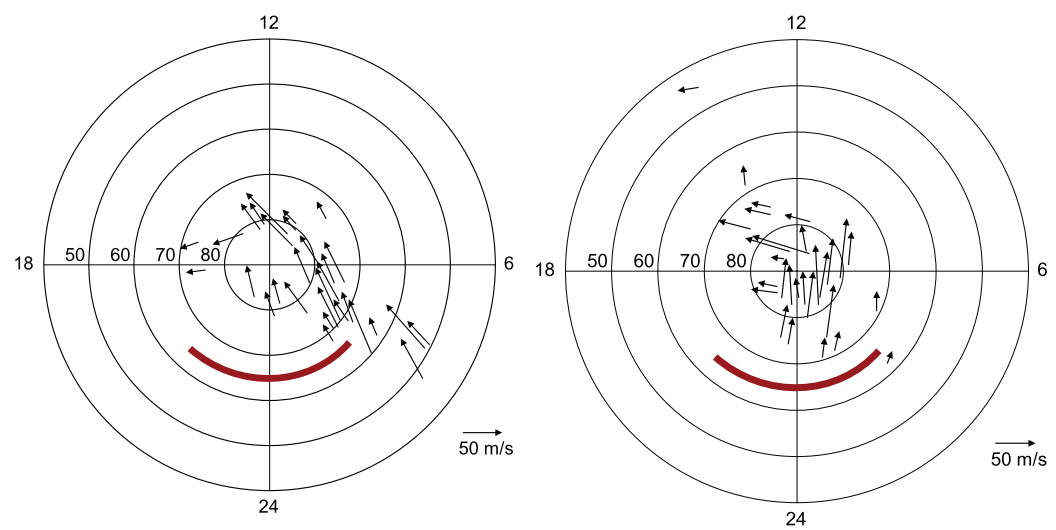

Figure 6. Directions of AGW propagation above the northern polar region for the polar day (left) and polar night (right) conditions.

equal when averaged over a period (Fedorenko, 2010). Let us write the average potential energy of an AGW in the form (Fedorenko, 2009)

$E_{p}=\frac{1}{4} \rho_{0}\left[v_{x}^{2}\left(\frac{\omega}{k_{x} c_{\mathrm{s}}}\right)^{2}+v_{z}^{2}\left(\frac{\omega_{\mathrm{b}}}{\omega}\right)^{2}\right]$,

where $v_{x}$ and $v_{z}$ are the norms of the horizontal and the vertical components of the particles' velocities. Since the average over a period kinetic energy of an AGW is equal to

$E_{k}=\frac{1}{4} \rho_{0}\left(v_{x}^{2}+v_{z}^{2}\right)$,

then, assuming $E_{p} \approx E_{k}$, it follows from Eqs. (3) and (4) that

$v_{x}^{2}+v_{z}^{2}=v_{x}^{2}\left(\frac{\omega}{k_{x} c_{\mathrm{s}}}\right)^{2}+v_{z}^{2}\left(\frac{\omega_{\mathrm{b}}}{\omega}\right)^{2}$

One can see from Eq. (5) that the extreme case $v_{x}=0$ yields $\omega=\omega_{\mathrm{b}}$ (Brunt-Väisälä oscillations), and the opposite case $v_{z}=0$ yields $\omega=k_{x} c_{\mathrm{s}}$ (Lamb mode).

DE2 data testify that both the horizontal and vertical components of velocities are non-zero and close to each other. Since $\omega \approx \omega_{\mathrm{b}}, u_{x}$ must tend to $c_{\mathrm{s}}$, according to Eq. (5). The classical AGW theory (Hines, 1960) prohibits the propagation of AGWs with such parameters in an isothermal atmosphere, since they fall into a restricted area in the $k_{x}$ vs. $\omega$ plot.

Thus, DE2 data analysis tends towards the existence of a dominant AGW mode in the polar thermosphere with $\omega \approx \omega_{\mathrm{b}}$ and $u_{x} \rightarrow c_{\mathrm{s}}$, which contradicts the classical theory.

\subsection{Wind control of AGWs}

The wind pattern in the polar thermosphere is due to the superposition of solar heating and the magnetospheric convection projected along the field lines. The spatial structure of this pattern is quite complex and the wind velocity can reach $300-1000 \mathrm{~m} \mathrm{~s}^{-1}$ depending on the geomagnetic activity (Crowley et al., 1989; Killeen et al., 1995).
The AGWs observed from a satellite can occur directly in the upper atmosphere, but they may also be connected to sources in the lower atmosphere. Using an extended gravity wave scheme (Yiğit et al., 2008) that accounts for realistic wave propagation and dissipation showed that lower atmospheric gravity waves propagate into the thermosphere and affect the general circulation of the high-latitude thermosphere under various conditions (Yiğit et al., 2009, 2012, 2014). Also, numerical simulations suggest that gravity waves propagate from the surface to the thermosphere (Hickey et al., 2009, 2010). In the polar ionosphere, there are very powerful sources of AGWs associated with particle precipitation and the dissipation of currents. Therefore, the AGWs caused by low atmospheric sources in the polar region are very difficult to distinguish. Phase velocities of observed AGWs exceed the speed of sound in the lower atmosphere. This probably indicates their upper atmospheric origin.

The directions of AGW propagation follow a systematic behaviour suggesting that their propagation is controlled by the wind (Fedorenko and Kryuchkov, 2011b). The most evident feature is the predominant propagation of AGW from the nightside to the dayside, i.e. towards the wind. During the polar day or the polar night the directions of AGW propagation follow the seasonal variation of the wind pattern in both hemispheres (Fedorenko and Kryuchkov, 2011b).

To illustrate this point we plotted in Fig. 6 the dominant directions of AGW propagation in the northern hemisphere for different UT and $K_{\mathrm{P}}$ values. Once can see that the AGW azimuths tend to follow the direction of Earth's rotation (counter-clockwise in the northern hemisphere). During the polar night (January-February) AGWs are tightly packed inside the polar cap and almost can not be detected outside the auroral oval. In this case the directions of AGW propagation are driven by the local polar wind pattern. During the polar day (June-July) an additional flow of AGWs towards the direction 14:00-15:00 LST can be seen. This flow is due to the wind caused by solar heating. On the nightside this flow escapes past the auroral oval, forming a tail-like struc- 
ture. On the dayside the AGW activity is constrained within the polar cap.

In the southern hemisphere this picture looks similar to the northern one. The main differences are more widely spread AGW azimuths and systematically lower AGW amplitudes. Apparently, this can be explained by a greater distance between the magnetic and the geographical poles in the south as compared to the north. The wind-driven circulation in the polar thermosphere can be considered as a superposition of two wind systems (Rees et al., 1983). One of them is the global wind circulation arising due to the absorption of solar UV- and EUV-radiation (tied to the geographic coordinate system). The second one is the vortex circulation arising from the convective motion of ionospheric plasma (tied to the geomagnetic coordinate system). The geomagnetic input introduces a strong UT-variation in thermospheric dynamics due to the offset of the geomagnetic poles from the geographic poles. The spatial area, disturbed by the polar thermospheric circulation, is greater in the southern hemisphere due to the greater distance between the geographic and geomagnetic poles. Accordingly, the region of increased wave activity associated with the wind in the southern hemisphere is also more extended.

Another clear indicator of AGW-wind coupling is the dependence of the AGW amplitude on the wind velocity (Fedorenko and Kryuchkov, 2011b). During AGW propagation, the density, pressure, temperature, and velocity are periodically changed. The amplitudes of these variables are connected to each other by the wave polarization relations. They are proportional to each other (Hines, 1960). We have analysed the variations of density, primarily because they were measured with greater precision and higher time resolution than the velocity and the temperature. Figure 7 shows the dependence of the AGW amplitude on the wind velocity to be almost linear. For an explanation of the functional form of this dependence needs further theoretical investigations. It should be noted that the waves with amplitudes below $1 \%$ are usually detected in mid-latitudes, and the waves with amplitudes over $2 \%$ are mostly seen in high latitudes.

The influence of the conductive ionosphere on the propagation of AGWs is significant at thermospheric altitudes. For further theoretical analysis of the interaction between AGW and the wind one needs also to consider the influence of the geomagnetic field on AGW properties (Kaladze et al., 2008; Khantadze et al., 2010).

\section{Implications for ground-based TID observations}

AGW propagation and dissipation are highly dependent on the background wind distribution. In the polar thermosphere, a large velocities of wind combined with narrow AGW intrinsic frequency band lead to a significant modification of the TID frequency spectrum observed from the ground. Due to the Doppler effect the apparent frequency $\Omega$ measured by

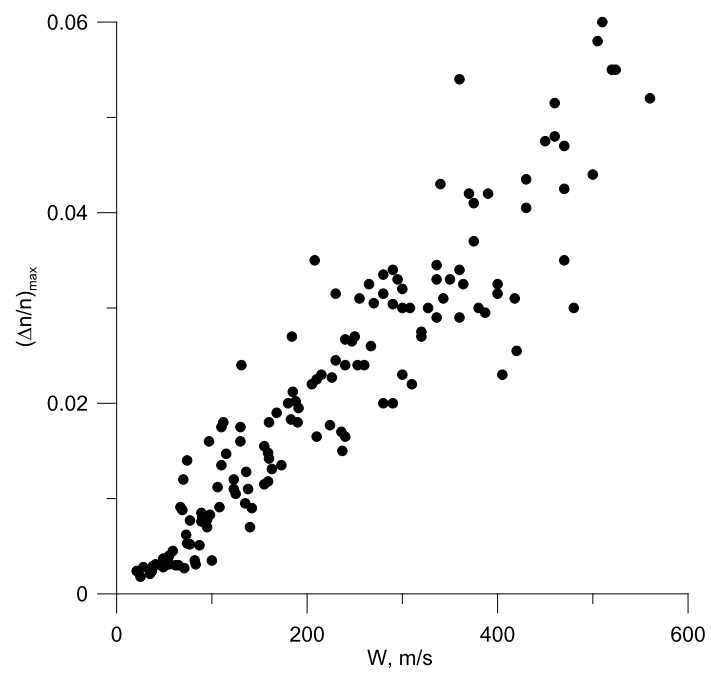

Figure 7. The dependence of AGW amplitude on the wind velocity.

a stationary observer in the ground frame equals

$\Omega=\omega+\boldsymbol{k}_{\mathrm{h}} \cdot \boldsymbol{W}=\omega+\left|\boldsymbol{k}_{\mathrm{h}}\right||\boldsymbol{W}| \cos \theta$,

where $\omega$ is the intrinsic frequency in the frame co-moving with the wind, $\boldsymbol{k}_{\mathrm{h}}$ is the horizontal projection of the wave vector, $\boldsymbol{W}$ is the wind velocity, and $\theta$ is the angle between the horizontal wave vector and the wind velocity.

Since AGWs propagate towards the wind, the scalar product $\boldsymbol{k}_{\mathrm{h}} \cdot \boldsymbol{W}$ is always negative and the apparent frequency is lower than the intrinsic frequency. According to Eq. (6), for AGWs with dominant horizontal wavelengths from 500 to $600 \mathrm{~km}$ and average wind velocities from 300 to $600 \mathrm{~m} \mathrm{~s}^{-1}$ typical for the polar thermosphere, the apparent periods measured on the ground should vary within the range from $30 \mathrm{~min}$ to $1 \mathrm{~h}$. The actually measured TID periods between $30 \mathrm{~min}$ and $70 \mathrm{~min}$ show good correspondence with this estimation.

Since the wind velocity in the polar thermosphere almost never gets below $250 \mathrm{~m} \mathrm{~s}^{-1}$, the minimum period detected on the ground should be about $20 \mathrm{~min}$. At perturbed geomagnetic conditions when the wind velocity exceeds $600 \mathrm{~m} \mathrm{~s}^{-1}$, the apparent TID periods can be as large as several hours, falling in the large-scale TID range.

\section{Conclusions}

The extensive statistical analysis of large volumes of DE2 data allowed us to determine the following properties of AGWs in the polar thermosphere:

1. AGWs are systematically observed in the thermosphere at 250 to $400 \mathrm{~km}$ above the polar caps regardless of the geomagnetic activity.

2. The intrinsic polar AGW frequency is close to the Brunt-Väisälä frequency. 
3. Polar AGWs systematically propagate towards the wind.

4. Polar AGW amplitudes do not depend on the altitude, but they almost linearly depend on the wind velocity.

The observed polar AGW properties listed above contradict the generally accepted hypothesis that AGWs are generated in the auroral regions. According to this hypothesis, the energy for AGWs is provided by the precipitating charged particles and the dissipation of polar current systems. This source is most effective at the altitudes about $100-120 \mathrm{~km}$, and should generate a broadband spectrum of AGWs. The periods of about several tens of minutes can be found in variations of the auroral electrojet and particle precipitation intensity and are regarded as potential sources of polar waves (Crowley and Williams, 1987; Williams et al., 1992).

Our results suggest that the polar waves are primarily driven by the thermospheric wind. One possible explanation for the listed polar AGW properties is that the primary waves from different sources are filtered by the non-uniformly moving medium due to the energy exchange between the waves and the medium (Lighthill, 2001). The dominant intrinsic AGW frequency close to the Brunt-Väisälä frequency can result from the evolution of the AGW spectrum in the horizontal shear flow (Didebulidze, 1999). Another possible explanation is that the inhomogeneous wind itself may generate AGWs, for example, due to the Kelvin-Helmholtz mechanism.

These dominant polar thermospheric waves are evident in the in situ measurements, but are very difficult to identify from the ground. This is caused by a strong spatial variability of the thermospheric wind pattern. The apparent TID frequency spectrum measured from the ground is substantially different from the intrinsic spectrum of polar AGWs due to the Doppler effect. An introduction of a correction for the wind velocity brings satellite and groundbased measurements to an agreement. The correction implies accounting the Doppler shift according to Eq. (6) of the measured frequency TID in comparison with the frequency of AGW, which is determined from satellite measurements. Typical TID periods of 30 to 70 min correspond to a Dopplershifted Brunt-Väisälä frequency assuming typical thermospheric wind velocities.

Since the pattern of the polar thermospheric winds are mostly tied to the geomagnetic frame, this should yield interesting observational consequences. The TIDs measured from the ground should manifest some effects, caused by the diurnal rotation of the Earth. In particular, both the amplitude and the period of TIDs should be maximal when the transpolar thermospheric wind current crosses the instrument's field of view.
Acknowledgements. The authors thank the "Second UK-Ukraine Meeting on Solar Physics and Space Science" organizers for support and Aleksei Parnowski for his help in the preparation of the manuscript.

Topical Editor J. Wild thanks T. Kaladze and one anonymous referee for their help in evaluating this paper.

\section{References}

Arbesser-Rastburg, B. and Jakowski, N.: Effects on Satellite Navigation, edited by: Bothmer, V. and Daglis, I., Space Weather Physics and Effects, Springer, Heidelberg, 383-402, 2007.

Carignan, G. R., Block, B. P., Maurer, J. C., Hedin, A. E., Reber, C. A., and Spencer, N. W.: The neutral mass Spectrometer on Dynamics Explorer, Space Sci. Instrum., 5, 429-441, 1981.

Cowling, D. H., Webb, H. D., and Yeh, K. C.: Group rays of internal gravity waves in a wind-stratified atmosphere, J. Geophys. Res., 76, 213-220, 1971.

Crowley, G. and Williams, P. J. S.: Observations of the source and propagation of atmospheric gravity waves, Nature, 328, 231233, 1987.

Crowley, G., Emery, B. A., Roble, R. G., Carlson, H. C., and Knipp, D. J.: Thermospheric dynamics during September 18-19, 19841. model simulations, J. Geophys. Res., 94, 16925-16944, 1989.

Didebulidze, G. G.: Evolution of Atmospheric Gravity Waves in Horizontal Shear Flow, Phys. Scr., 60, 593-600, 1999.

Dudis, J. J. and Reber, C. A.: Composition effects in thermospheric gravity waves, Geophys. Res. Lett., 3, 727-730, 1976.

Fedorenko, A. K.: Recovery of Characteristics of Atmospheric Gravity Waves in Polar Regions Based on Mass Spectrometric Satellite Measurements, Radio Phys. Radio Astron., 14, $254-$ 265, 2009.

Fedorenko, A. K.: Energy Balance of Acoustic Gravity Waves above the Polar Caps according to the Data of Satellite Measurements, Geomagn. Aeron., 50, 107-118, 2010.

Fedorenko, A. K. and Kryuchkov, E. I.: Distribution of MediumScale Acoustic Gravity Waves in Polar Regions According to Satellite Measurement Data, Geomagn. Aeron., 51, 520-533, 2011a.

Fedorenko, A. K. and Kryuchkov, E. I.: Wind-induced Effects in the Parameters of Atmospheric Gravity Waves in the Polar Ionosphere, Radio Phys. Radio Astron., 2, 315-323, 2011 b.

Förster, M. and Jakowski, N.: Geomagnetic storm effects on the topside ionosphere and plasmasphere: A compact tutorial and new results, Surv. Geophys., 21, 47-87, 2000.

Hickey, M. P., Schubert, G., and Walterscheid, R. L.: Propagation of tsunami-driven gravity waves into the thermosphere and ionosphere, J. Geophys. Res., 114, A08304, doi:10.1029/2009JA014105, 2009.

Hickey, M. P., Walterscheid, R. L., and Schubert, G.: Wave mean flow interactions in the thermosphere induced by a major tsunami, J. Geophys. Res. Space Phys., 115, A09309, doi:10.1029/2009JA014927, 2010.

Hines, C. O.: Internal gravity waves at ionospheric heights, Can. J. Phys., 38, 1441-1481, 1960.

Johnson, F. S., Hanson, W. B., Hodges, R. R., Coley, W. R., Carignan, G. R., and Spencer, N. W.: Gravity waves near $300 \mathrm{~km}$ over the polar caps, J. Geophys. Res., 100, 23993-24002, 1995. 
Kaladze, T. D., Pokhotelov, O. A., Shah, H. A., Khan, M. I., and Stenflo, L.: Acoustic-gravity waves in the Earth's ionosphere, J. Atmos. Sol.-Terr. Phys., 70, 1607-1616, 2008.

Khantadze, A. G., Jandieri, G. V., Ishimaru, A., Kaladze, T. D., and Diasamidze, Zh. M.: Electromagnetic oscillations of the Earth's upper atmosphere (review), Ann. Geophys., 28, 13871399, doi:10.5194/angeo-28-1387-2010, 2010.

Killeen, T. L., Won, Y. I., Nicieyewski, R. J., and Burns, A. G.: Upper thermosphere winds and temperatures in the geomagnetic polar cap: Solar cycle, geomagnetic activity, and interplanetary magnetic fields dependencies, J. Geophys. Res., 100, 2132721342, 1995.

Lighthill, J.: Waves in Fluids, Cambridge University Press, New York, 2001.

Rees, D., Fuller-Rowell, T. J., Gordon, R., Killeen, T. L., Hays, P. B., Wharton, L. E., and Spencer, N. W.: A comparison of the wind observations from the Dynamics Explorer satellite with the predictions of a global time-dependent model, Planet. Space Sci., 31, 1299-1314, 1983.

Spencer, N. W., Wharton, L. E., Niemann, H. B., Hedin, A. E., Carignan, G. R., and Maurer, J. C.: The Dynamics Explorer wind and temperature spectrometer, Space Sci. Instrum., 5, 417-428, 1981.
Vincent, R. A. and Eckermann, S. D.: VLF radar observations of mesoscale motions in the troposphere: Evidence for gravity wave Doppler shifting, Radio Sci., 25, 1019-1037, 1990.

Williams, P. J. S., Lewis, R. V., Virdi, T. S., Lester, M., and Nielsen, E.: Plasma flow bursts in the auroral electrojets, Ann. Geophys., 10, 835-848, 1992, http://www.ann-geophys.net/10/835/1992/.

Yiğit, E., Aylward, A.D., Medvedev, A.S.: Parameterization of the effects of vertically propagating gravity waves for thermosphere general circulation models: Sensitivity study, J. Geophys. Res., 113, D19106, doi:10.1029/2008JD010135, 2008.

Yiğit, E., Medvedev, A. S., Aylward, A. D., Hartogh, P., and Harris, M. J.: Modeling the effects of gravity wave momentum deposition on the general circulation above the turbopause, J. Geophys. Res., 114, D07101, doi:10.1029/2008JD011132, 2009.

Yiğit, E., Medvedev, A. S., Aylward, A. D., Ridley, A. J., Harris, M. J., Moldwin, M. B., and Hartogh, P.: Dynamical effects of internal gravity waves in the equinoctial thermosphere, J. Atmos. Sol.-Terr. Phys., 90-91, 104-116, 2012.

Yiğit, E., Medvedev, A. S., England, S. L., and Immel, T. J.: Simulated variability of the high-latitude thermosphere induced by small-scale gravity waves during a sudden stratospheric warming, J. Geophys. Res., 119, 357-365, 2014. 